Thorax (1973), 28, 399.

\title{
Control of massive cardiac haemorrhage by balloon catheter
}

\author{
P. DUBE \\ Queen Elizabeth Hospital, Birmingham
}

\begin{abstract}
The use of a balloon catheter to achieve the control of haemorrhage from the chambers of the heart has not been described previously. A case is reported in which this method was used in an emergency situation. It has been tested successfully, as a planned procedure, during four closed mitral valvotomy operations.

Balloon catheters have been used in vascular surgery for some time. Taylor and Williams (1962) used a Foley's catheter to prevent haemorrhage from an arteriovenous fistula at the time of its repair. Fogarty et al. (1963) described the use of Fogarty's catheter during extraction of arterial emboli and thrombi. Control of hepatic venous bleeding by placing a balloon catheter in the inferior vena cava in seven dogs was described by Doty and Berman (1970), and Foster, Morgan, and Threlkel (1971) used balloon catheters to achieve proximal control of the abdominal aorta. Thomas (1971) described the use of Fogarty catheters and Bakes common bile duct dilators to control bleeding during operation in complicated peripheral vascular lesions. Arbulu and Thomas (1972) reported a case of a gunshot wound in which haemorrhage from the inferior vena cava close to the heart was controlled by using a Foley's catheter.
\end{abstract}

This paper describes a method for controlling bleeding from the chambers of the heart. Such bleeding is often massive and rapid and may lead to the death of the patient.

Patients most likely to bleed are those who have had previous heart surgery. In some cases the previous operation seems to make the wall of the cardiac chambers more friable and stitches cut through more easily.

\section{CASE REPORT}

D.L.G., a woman of 48 years who had had a closed mitral valvotomy for mitral stenosis in 1956, complained of increasing shortness of breath for three years and recent attacks of paroxysmal nocturnal dyspnoea. Examination revealed the signs typical of mitral re-stenosis. In September 1971 posterolateral thoracotomy was performed through the previous incision. The pericardium was densely adherent to the heart. The pericardium was separated by sharp dissection. Slight bleeding from the left atrium was controlled by suturing. Extensive calcification and the friable nature of the left atrial wall made an approach through this area unsafe. It was decided, therefore, to explore the valve through the superior pulmonary vein. The vein was dissected and a longitudinal incision was made in the vein after inserting two stay sutures on either side of the proposed incision. A finger was inserted into the left atrium. At this time the junction of the vein with the left atrium started to split causing bleeding. Suturing and an attempt to apply a clamp made the situation worse. The pulmonary side of the vein was occluded by a tape. By this time the bleeding was considerable and the blood pressure had fallen to $50 \mathrm{mmHg}$ systolic. A Foley's catheter 16 F.G. with a $30 \mathrm{ml}$ balloon was inserted into the left atrium through the incision in the pulmonary vein and the balloon was inflated with $25 \mathrm{ml}$ saline. Gentle traction controlled the bleeding. At this time the heart was beating adequately with a systolic blood pressure of $50 \mathrm{mmHg}$. Unfortunately, before anything else could be done, a calcified spike burst the balloon, resulting in further haemorrhage. The catheter was replaced promptly. During this period the heart arrested as a result of the further sudden haemorrhage and remained so despite all attempts to restart it. Throughout the period of cardiac massage the balloon catheter maintained adequate control of the haemorrhage.

\section{DISCUSSION}

Severe haemorrhage during closed mitral valvotomy occurs rarely. Calcified thrombus in the left atrium is occasionally seen and was the cause of the balloon bursting with further severe haemorrhage and cardiac arrest, terminating in the death of the patient.

Effective control of the haemorrhage from the left atrium by a balloon catheter was achieved 
in this case. Balloon tamponade was tried successfully during four uncomplicated closed mitral valvotomy operations. In each case complete and effective control of blood loss was achieved by the balloon plug. A large Fogarty catheter with a strong balloon will achieve the same objective and may well be more effective in the control of ventricular bleeding than the Foley's catheter.

It is suggested that for the atrial haemorrhage a Foley's catheter of a large size (22-28 F.G.) with a $30 \mathrm{ml}$ balloon inflated with sterile normal saline be used. If air is used to inflate the balloon, bursting may produce air embolism. A second catheter should be kept available in case the first balloon is damaged. Once tamponade has been achieved, the cardiovascular state should be stabilized.

The Foley's catheter can be used for blood transfusion directly into the heart chamber by connecting the tubing of a sterile drip set to the catheter. It is essential to ensure that there is no air in the drip system before the transfusion begins. It is also desirable to use a blood warmer for such rapid transfusion.
The author wishes to thank the surgeons at the East Birmingham and Queen Elizabeth Hospitals, Birmingham, under whose care the patients were admitted.

\section{REFERENCES}

Arbulu, A., and Thomas, N. W. (1972). Control of bleeding from a gunshot wound of the inferior vena cava at its junction with the right atrium by means of a Foley $\vec{x}$ catheter. J. thorac. cardiovasc. Surg., 63, 427.

Doty, D. B., and Berman, I. R. (1970). Control of hepatic $\infty$ venous bleeding by transvenous balloon catheter. Surg. Gynec. Obstet., 131, 449.

Fogarty, T. J., Cranley, J. J., Krause, R. J., Strasser, E. S., \& and Hafner, C. D. (1963). A method for extraction of $\circ$ arterial emboli and thrombi. Surg. Gynec. Obstet., 116, 241.

Foster, J. H., Morgan, C. V., and Threlkel, J. B. (1971). Proximal control of aorta with a balloon catheter. Surg. Gynec. Obstet., 132, 693.

Taylor, H., and Williams, E. (1962). Arteriovenous fistula following disk surgery. Brit. J. Surg., 50, 47.

Thomas, T. V. (1971). Control of bleeding in complicated peripheral vascular lesions. Surg. Gynec. Obstet., 132, 1015. 